\title{
Anti-CD123/CD3 Bispecific Antibody APVO436
}

National Cancer Institute

\section{Source}

National Cancer Institute. Anti-CD123/CD3 Bispecific Antibody APVO436. NCI Thesaurus. Code C157257.

An immunoglobulin Fc-modified bispecific monoclonal antibody against the tumorassociated antigen (TAA) CD123 and the human T-cell surface antigen CD3 bispecific monoclonal antibody, with potential immunostimulatory and antineoplastic activities. Upon administration, anti-CD123/CD3 monoclonal antibody APV0436 simultaneously binds to both CD3-expressing T-cells and CD123-expressing cancer cells, thereby crosslinking CD123-expressing tumor cells and cytotoxic T-lymphocytes (CT LS). This results in the activation and proliferation of T-cells and causes CTL-mediated cell lysis of CD123-expressing tumor cells. CD123, the interleukin-3 receptor alpha chain, is overexpressed in a variety of hematological malignancies; its expression is low or absent in normal hematopoietic progenitors and stem cells. The Fc domain on the antibody prolongs the half-life of the bispecific antibody. Compared to some other CD123 x CD3 targeting bispecific antibodies, APVO436 causes less cytokine release upon T-cell stimulation. 